\title{
Quantitative, Super-resolution Localization of Small RNAs with sRNA-PAINT
}

Kun Huang ${ }^{1}$, Feray Demirci ${ }^{2}$, Blake Meyers ${ }^{3}$ and Jeffrey Caplan ${ }^{4}$

${ }^{1}$ University of Delaware, Newark, Delaware, United States, ${ }^{2}$ FiDoSoft Software Consulting, Redmond, Washington, United States, ${ }^{3}$ Donald Danforth Plant Science Center, St. Louis, Missouri, United States, ${ }^{4}$ Delaware Biotechnology Institute, Newark, Delaware, United States

Small RNAs are 21- to 24-nt non-coding RNAs that play important regulatory roles in the life of both animals and plants. Their small size and high diversity had made it challenging to develop methods that have sufficient resolution and specificity. We created a method sRNA-PAINT, for the detection of small RNA within nanometer resolution. Our method utilizes resolution and quantification advances in DNAPAINT (DNA-based points accumulation in nanoscale topography) methodologies, and combines the specificity of locked nucleic acid (LNA) in situ detection of small RNAs. The probe design is the most critical step of the sRNA-PAINT method, and therefore, we created an online tool called VARNISH (Vetting \& Analysis of RNA for in situ Hybridization probes) for automated design of sRNA-PAINT probes (https://wasabi.ddpsc.org/ apps/varnish/). sRNA-PAINT decouples the blinking events from dye photophysics, using the binding and dwelling kinetics of a short dye-labeled oligonucleotide "imager strand" to localize target-specific "docking strands". qPAINT from the Picasso software can be applied to sRNA-PAINT images for counting molecules by analyzing the predictable and programmable binding kinetics of the imager strand to the docking strand. Highly-multiplexed, sequential detection of sRNAs can be done by multiplex VARNISH probes with different docking strand combinations. We applied sRNA-PAINT for detecting and quantifying small RNAs in different cell layers of early developmental stage maize anther that are important for male sexual reproduction. We confirmed that target small RNAs localized to the tapetum layer and pollen mother cells. Using sRNA-PAINT, which has the resolution limit down to $10 \mathrm{~nm}$, our results showed subcellular localization of target sRNAs at the single molecule level. With the ability to count the exact copy number, this method could be used to investigate many biological questions that require precise subcellular localization or quantitative data, from small RNA biogenesis to the function of sRNAs. 

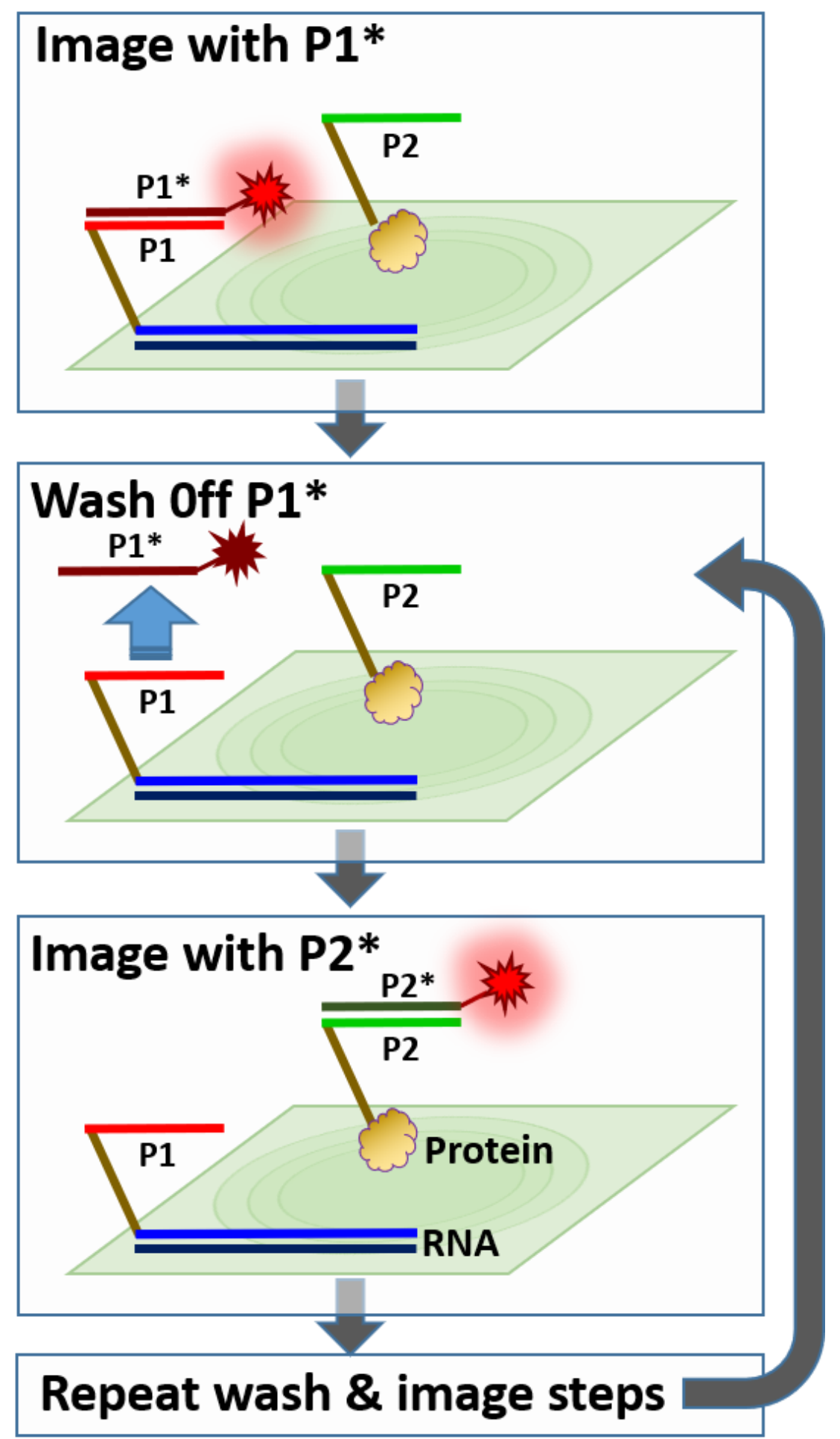

Figure 1. Multiplexing RNA-PAINT. RNA-PAINT can be multiplexed by modifying and combining with an approach called "Exchange-PAINT". Multiple RNA-PAINT or exchange-PAINT probes with different docking strands (i.e. P1, P2,...PN) will be hybridized to samples. The first probe will be imaged with the $\mathrm{P} 1 *$ imager. The $\mathrm{P} 1 *$ imager will be washed off and then the sample will be imaged with $\mathrm{P} 2 *$ imager. The wash and image steps can be repeated sequentially for multiplexing. 

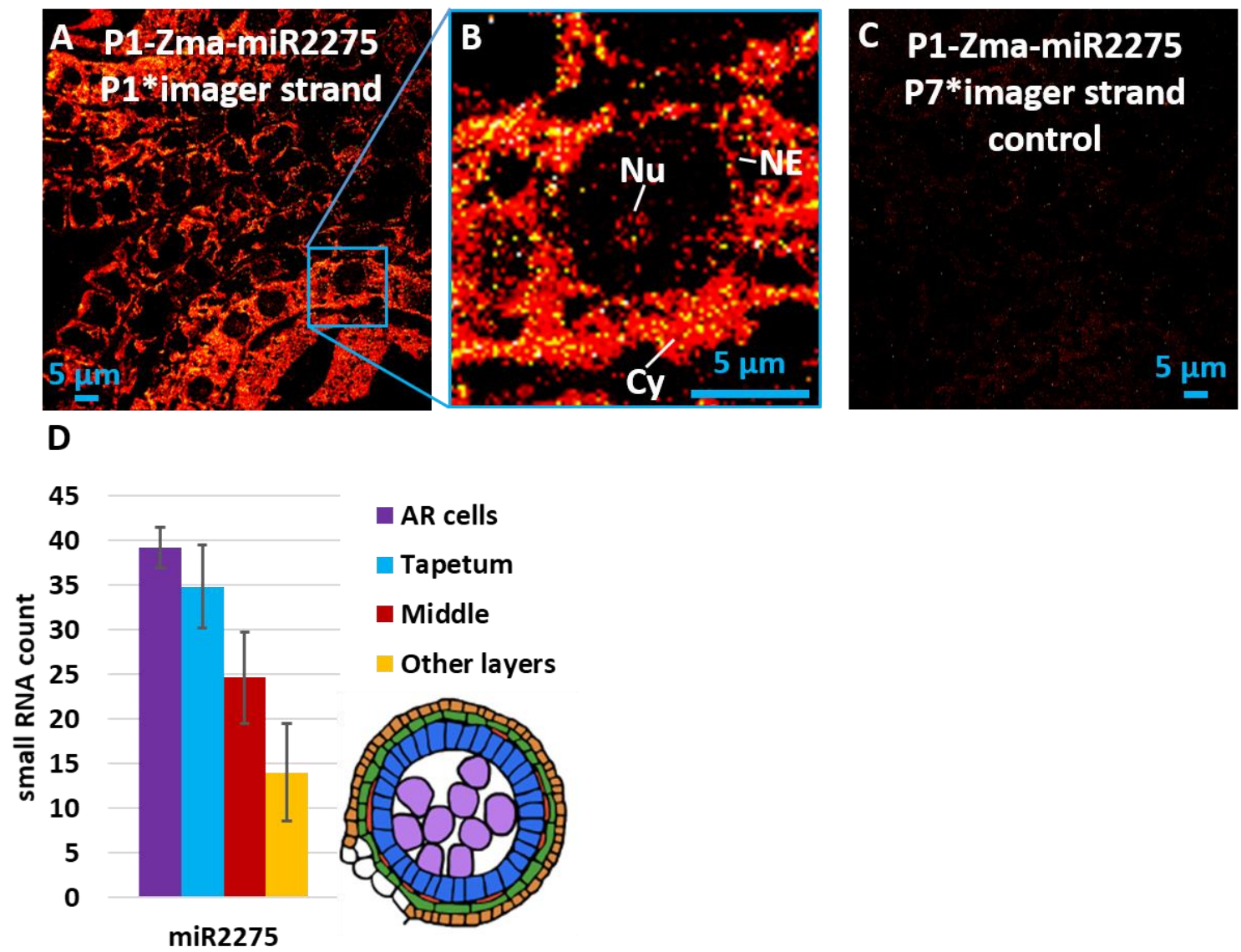

Figure 2. RNA-PAINT of sRNA in maize anther. (A) RNA-PAINT using a P1 docking strand linked to a LNA probe for Zma-miR2275. Specific signal in the expected tissue layers was observed when the corresponding P1* imager strand. (B) High magnification of boxed area showing localization around nucleolus $(\mathrm{Nu})$, along the nuclear envelope (NE), and in the cytosol (Cy). (C) Scant signal was detected with the non-specific P7* control. (D) Copy number of miR2275 in each anther cell layer.

\section{References}

Huang, K. et al. Quantitative, super-resolution localization of small RNAs with sRNA-PAINT. bioRxiv, 716696 (2019).

Jungmann, R. et al. Multiplexed 3D cellular super-resolution imaging with DNA-PAINT and ExchangePAINT. Nature Methods 11 (2014).

Zhai, J.X. et al. Spatiotemporally dynamic, cell-type-dependent premeiotic and meiotic phasiRNAs in maize anthers. PNAS 112 (2015). 